\title{
Coherent Raman Scattering microscopy: Technology developments and biological applications
}

\author{
I. Pope, F. Masia*, J. Bradley, K. Ewan, A. Karuna, L. Payne, I. Guschina, J. Harwood, K. Swann, \\ T. Dale, P. Watson, W. Langbein* and P. Borri \\ Cardiff University School of Biosciences, Museum Avenue, Cardiff CF10 3AX, United Kingdom \\ * Cardiff University School of Physics and Astronomy, The Parade, Cardiff CF24 3AA, United Kingdom \\ e-mail: borrip@cf.ac.uk
}

\begin{abstract}
Optical microscopy is an indispensable tool that is driving progress in cell biology, and is still the only practical means of obtaining spatial and temporal resolution within living cells and tissues. Coherent Raman Scattering (CRS) microscopy has emerged in the last decade as a powerful multiphoton microscopy technique which overcomes the need for fluorescent labelling and yet retains biomolecular specificity and intrinsic 3D resolution. We have developed several home-built CRS microscopes featuring innovative excitation/detection schemes and quantitative image analysis. Our second-generation multimodal CRS instrument is based on a single 5fs Ti:Sa laser source with 350nm bandwidth capable of exciting a wide vibrational range from $1200 \mathrm{~cm}^{-1}$ to $3800 \mathrm{~cm}^{-1}$, thus enabling hyperspectral microscopy. Alongside CRS, it also features simultaneous detection of two-photon fluorescence and second harmonic generation for correlative studies. Our latest technology developments include high-content high-throughput label-free quantitative chemical imaging via Bessel beam illumination and sparse sampling. Our latest biological applications feature quantitative imaging of i) intracellular lipid droplets in live mouse oocytes and early embryos, ii) cancer organoids and their responses to drugs, iii) freshwater microalgae as potential food supplements for omega- 3 fatty acids.
\end{abstract}

Keywords: vibrational microscopy, coherent Raman scattering, multiphoton microscopy, bioimaging.

\section{INTRODUCTION}

Coherent Raman scattering microscopy has emerged in the last two decades as a powerful label-free chemically specific multiphoton microscopy technique [1]. In Raman scattering, the interaction of light with vibrating chemical bonds gives rise to light at a different wavelength compared to the incident field. The wavelength shift is a direct signature of the frequency of the vibration which in turn depends on the type of chemical bond. This scattering phenomenon usually produces a very weak signal, but can be strongly enhanced in CRS when two short laser pulses (denoted as pump and Stokes of optical frequencies $\omega_{\mathrm{p}}$ and $\omega_{\mathrm{s}}$ respectively) are used to coherently drive the vibrations at frequency $\omega_{\mathrm{p}}-\omega_{\mathrm{s}}$, such that Raman scattered light from coherently driven molecular vibrations in the focal volume constructively interferes. For applications in cell biology, CRS microscopy has attracted a lot of interest as a label-free yet chemically specific alternative to fluorescence microscopy, overcoming the drawbacks of labelling artefacts, sample manipulation, photobleaching and phototoxicity. CRS imaging of living cells with high spatial and temporal resolution has been demonstrated on many biological samples, with particular emphasis on imaging lipids owing to the large number of identical $\mathrm{CH}$ bonds in their acyl chains [1]. Initial demonstrations of CRS microscopy focused on showing high-speed imaging up to video rate but with limited chemical information due to the detection of a single $\mathrm{CH}$-stretch vibrational resonance being unable to provide a quantitative chemical map of the lipid concentration. Subsequent implementations of multiplex or hyperspectral CRS imaging, where a vibrational spectrum is measured for each spatial point, enabled much higher specificity and quantitative spatiotemporal chemical profiling. Notably, substantial progress has been made in the quantitative data analysis associated with hyperspectral imaging when using coherent anti-Stokes Raman scattering (CARS) acquisition [2]. The ability to measure the vibrationally nonresonant part of the third order susceptibility in CARS enables straightforward in situ calibration via ratiometric comparison with a standard nonresonant medium in the sample (e.g., water or the glass coverslip), which in turn results in the quantitative retrieval of susceptibility spectra factorized into several chemical components with physically meaningful concentration units.

We have developed several home-built CRS microscopes featuring innovative excitation/detection schemes and quantitative image analysis. Here, we show our latest advances in terms of technology developments and applications to cell biology.

\section{TECHNOLOGY DEVELOPMENTS}

Our second-generation CARS instrument is based on a single 5fs Ti:Sa laser source with $350 \mathrm{~nm}$ bandwidth capable of exciting a wide vibrational range from $1200 \mathrm{~cm}^{-1}$ to $3800 \mathrm{~cm}^{-1}$, thus enabling hyperspectral microscopy [3]. To achieve high-spectral resolution with a broadband source, we use spectral focusing in which an equal linear chirp is applied to both the pump and Stokes beams (via glass blocks of known dispersion [4]), 
resulting in a constant instantaneous frequency difference (IFD), which can be tuned by varying the delay between pump and Stokes beams. Alongside CARS, the system also features simultaneous detection of twophoton fluorescence and second harmonic generation (in forward and epi geometry) for correlative multimodal studies, using a home-built detector assembly with appropriate filters and photomultipliers [3]. The microscope stand is equipped with a range of objectives with variable numerical aperture (from 0.75NA dry to 1.45NA oil) and corresponding condensers. It is also equipped with standard wide-field fluorescence, DIC imaging and an environmental chamber for mammalian live cell studies at $37^{\circ} \mathrm{C}$ in a humidified $5 \% \mathrm{CO} 2$ atmosphere.

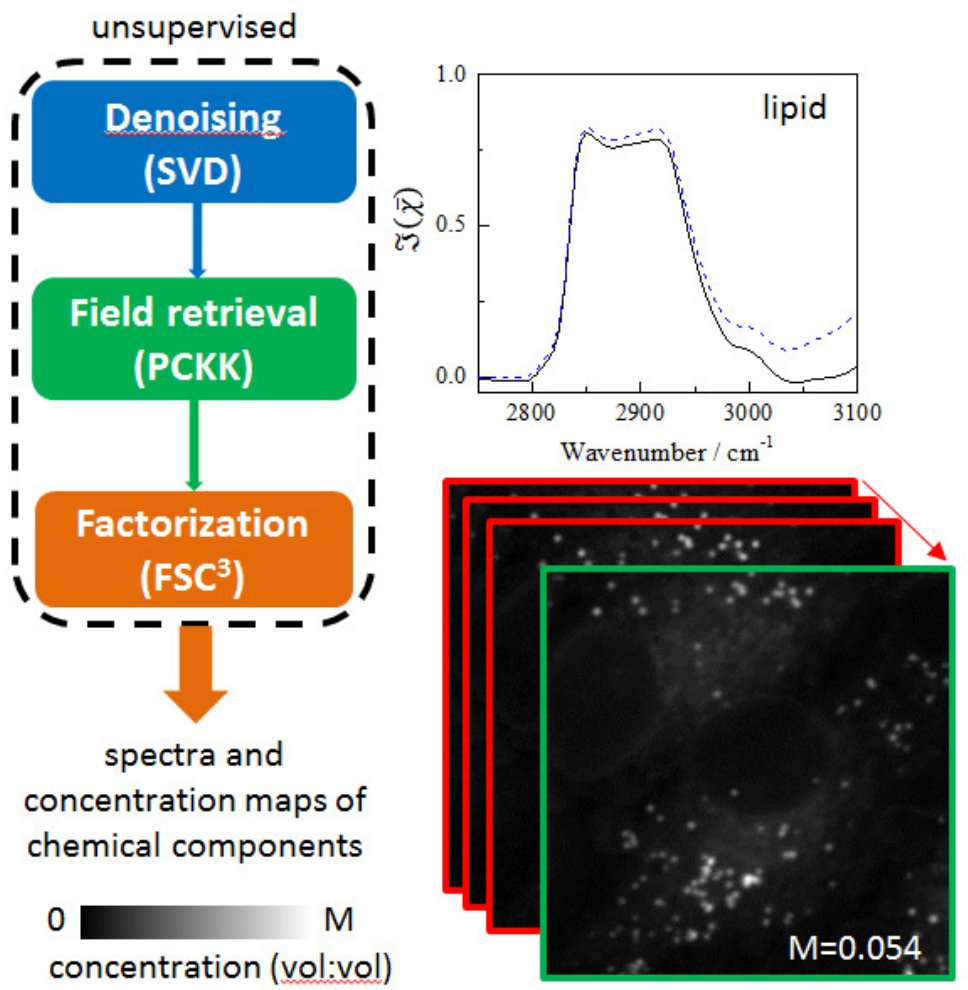

Gauss z-stack weighted average

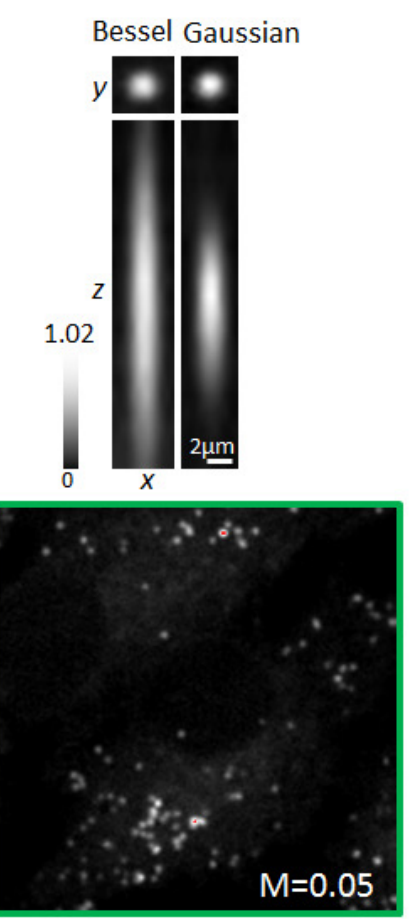

Bessel single z

Figure 1.Left: Sketch of the quantitative data analysis pipeline developed to retrieve susceptibility spectra and concentrations of chemical components (see text and [2]). Right: Lipid component in HeLa cells; comparison between Gaussian and Bessel beam illumination. The spatial distribution of lipid droplets is equally resolved, demonstrating the ability of Bessel beam excitation to reduce image acquisition without losing information on lateral organization, by z-averaging over the entire cell height in a single scan. Top right shows the concentration of a polystyrene bead of 1 um nominal size in agar (see text and [4]).

To quantitatively analyse hyperspectral CARS data, we have developed a powerful data analysis algorithm, which we named "Factorization into Susceptibilities and Concentrations of Chemical Components" (FSC ${ }^{3}$ ) [2]. The pipeline of the analysis is schematically shown in Fig.1. Briefly, hyperspectral CARS images are noisefiltered with a singular value decomposition (SVD) algorithm on the square root of the CARS intensity to retain only components above noise. CARS intensity ratios are calculated by dividing the background corrected CARS intensity by the corresponding nonresonant CARS intensity measured in glass under the same excitation and detection conditions. The phase-corrected Kramers-Kronig method (PCKK) is used to retrieve from the CARS intensity ratio the complex CARS third-order susceptibility (normalized to the nonresonant value in glass), which is linear in the concentration of chemical components. We then used non-negative matrix factorization applied to the imaginary part and the average real part of the susceptibility with an additional concentration constraint (FSC ${ }^{3}$ method) to obtain susceptibility spectra of independently varying chemical components and their volume concentrations. Notably, the $\mathrm{FSC}^{3}$ is unsupervised; that is, it does not require prior knowledge of the spectra of chemical components.

Most recently, we have extended the instrument capabilities by adding the option of Bessel beam illumination, to extend the axial depth of field, with a hyperspectral sparse sampling approach [4]. The motivation for this development is to enable screening of multiwell plates at high speed while retaining the highcontent chemical analysis of hyperspectral imaging. Specifically, the rationale for using a Bessel beam is to provide a nonlinear probe volume with an axial extension encompassing the full thickness of a $2 \mathrm{D}$ cell culture (see Fig.1) in a multiwell plate while retaining high confinement in-plane, thus enabling the sample's lateral organization to be probed at high resolution while averaging axially for fast acquisition. 


\section{BIOLOGICAL APPLICATIONS}

We are working on a wide range of biological applications, including stem cell adipogenesis [5], lipid droplets and metabolism in live mouse oocytes and early embryos [6], identifying label-free markers of cell types in cancer organoids and their responses to drugs, studying freshwater microalgae as potential food supplements for omega-3 fatty acids (FAs). The latter two are schematically shown in Fig.2 and 3 respectively. Organoids are 3D microtissues that recapitulate much of the morphology of the tissues from which they are derived. Notably, it has been shown that patient-derived cancer organoids retain cellular heterogeneity that matches that of the patient tumours. However, these 3D cell systems have not yet become routine tools in pharmaceutical discovery because the complex environments they recreate are not matched by robust quantitative and scalable analysis methods. CARS microscopy has the potential to provide a quantitative labelfree analysis tool of live organoids in 3D, removing the limitations associated with current staining and fixation protocols. Fig. 2 shows our spatially-resolved chemical analysis of a live metastatic human colorectal cancer organoid using hyperspectral CARS and $\mathrm{FSC}^{3}$. We find chemical components that allow us to distinguish the cell nuclei (blue) from the cytosol (green) and the lipid membranes (red) in a completely label-free way. We are currently investigating the potential of this methodology to identify markers that can be used to differentiate cell types and their responses to drugs.
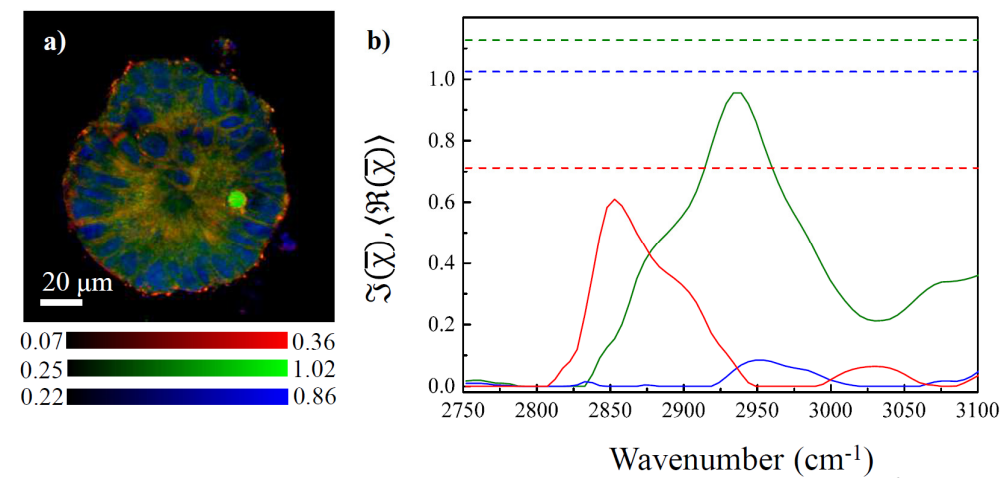

Figure 2. FSC ${ }^{3}$ analysis of a metastatic human colorectal cancer organoid. FSC concentration maps (a) and their spectra (b) are shown, colour coded.

Omega-3 FAs are known to be essential in the healthy human development (for example of the brain and the eye), and to play an important role in the prevention and treatment of a number of very important inflammatory conditions including ischemic heart disease. To act physiologically, these FAs need to be $20 \mathrm{C}$ or $22 \mathrm{C}$ in length and most dietary advice is to consume significant quantities of such long chain polyunsaturated fatty acids (PUFAs). At present, long chain omega-3 PUFAs are provided for human consumption mainly in the form of fish oils, which is expensive for continental countries. Moreover, overfishing is a growing global concern for the sustainability of the environment. Since the primary source of these PUFAs is microalgae, they are a very promising target for research into low-cost alternatives to oily fish. Notably, microalgae can produce high amounts of long chain PUFAs, in contrast to land plants, especially in response to different growth conditions. Fig.3 shows the potential of label-free CARS microscopy to image the lipid content and its spatial distribution in two strains of algae (Chlorella sorokiniana and Myrmecia bisecta), despite the large autofluorescence background from pigments in these species. With this technique, we are currently investigating how the accumulation of omega-3 fatty acids is manifesting at the sub-cellular level in living microalgal strains, and how it can be increased by altering their growth conditions (e.g. nitrogen or phosphorus limitation).

\section{CONCLUSIONS}

We have shown our latest advances in label-free coherent Raman scattering microscopy in terms of instrument technology development, quantitative data analysis, and biological applications. Particularly promising is the use of a single source broadband femtosecond laser with spectral focussing for hyperspectral CARS combined with our quantitative data analysis pipeline which retrieves Raman-like susceptibility spectra and factorizes them into chemical components with associated concentration maps in vol/vol units. With the addition of a Bessel beam illumination and a sparse sampling approach, the technique now reaches acquisition speeds that make CARS imaging within a multi-well format for high-throughput screens achievable. With this powerful technology we are investigating a wide variety of biological questions spanning from developmental biology, to cancer research to global challenges in food supply. 

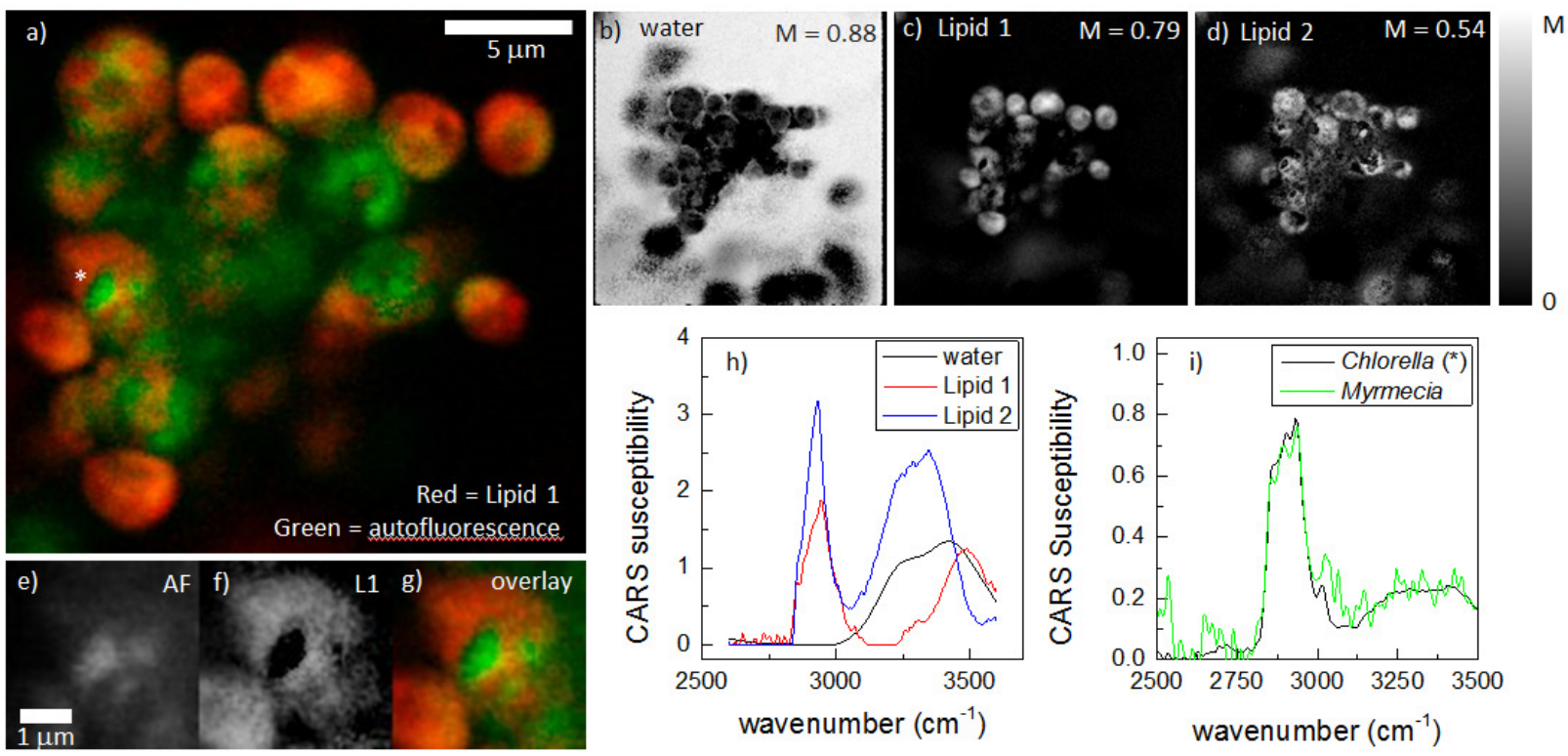

Figure 3. Hyperspectral CARS microscopy on living cells from Chlorella sorokiniana and Myrmecia bisecta after 2 weeks cultivation. a) 2D xy image showing an overlay of the spatially-resolved map of the lipid concentration (L1) and autofluorescence $(A F)$ in Chlorella sorokiniana. b-c) Concentration maps in vol:vol units from 0 to $M$. Corresponding spectra suggesting two lipid types shown in (h). e-g) zoom over the region (indicated by *) of the AF and L1 maps, and corresponding overlay. i) Single-point spectra showing different lipid types in the two species, consistent with Chlorella sorokiniana being rich in $\alpha$-linolenic acid (18:3n-3) and Myrmecia bisecta having a high arachidonic acid (20:4n-6) content, hence higher $=C H$ band at $3010 \mathrm{~cm}^{-1}$.

\section{ACKNOWLEDGEMENTS}

This work was funded by the U.K. BBSRC Research Council (grant no. BB/H006575/1 and BB/P007511/1), the EPSRC Research Council (EP/I005072/1), Cancer Research UK (grant no.C368/A22099), and the Royal Society (grant no. CH160031 and WM140077).

\section{REFERENCES}

[1] A. Zumbusch, W. Langbein, and P. Borri: Nonlinear vibrational microscopy applied to lipid biology, Progress in Lipid Research vol. 52, pp. 615-632, 2013.

[2] F. Masia et al.: Quantitative chemical imaging and unsupervised analysis using hyperspectral coherent anti-Stokes Raman scattering microscopy, Analytical Chemistry vol.85, pp. 10820-10828, 2013.

[3] I. Pope et al.: Simultaneous hyperspectral differential-CARS, TPF and SHG microscopy with a single 5 fs Ti:Sa laser, Optics Express 21, pp. 7096-7106, 2013.

[4] F. Masia et al.: Bessel-beam hyperspectral CARS microscopy with sparse sampling: Enabling high-content high-throughput label-free quantitative chemical imaging, Analytical Chemistry vol. 90, pp. 3775-3785, 2018.

[5] C. Di Napoli et al.: Quantitative spatio-temporal chemical profiling of individual lipid droplets by hyperspectral CARS microscopy in living human adipose-derived stem cells, Analytical Chemistry vol. 88, pp. 3677-3685, 2016.

[6] J. Bradley et al:: Quantitative imaging of lipids in live mouse oocytes and early embryos using CARS microscopy, Development vol. 143, pp. 2238-2247, 2016. 